\title{
CHRONIC GRANULOMATOUS MASTITIS: CASE REPORT
}

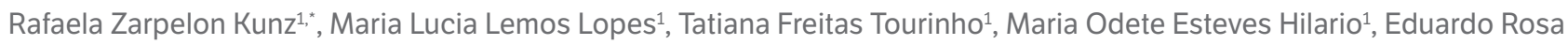
de Oliveira ${ }^{1}$, Thiago Willers ${ }^{1}$, Rafael Coradin ${ }^{1}$, Gilberto Scanagatta ${ }^{1}$, Raissa Velasques de Figueiredo ${ }^{1}$, Gabriela Sasso Padilha $^{1}$, Bruno Trevisann ${ }^{1}$, Luana Ribeiro Carlos ${ }^{1}$, Lilian Scussel Lonzetti ${ }^{1}$

1. Universidade Federal de Ciências da Saúde de Porto Alegre, Porto Alegre (RS), Brasil.

*Corresponding author: consultoriowillers@gmail.com

\section{BACKGROUNG}

Idiopathic granulomatous mastitis is a benign chronic inflammatory disease, with unknown etiology. It presents with clinical and radiological changes suggestive of inflammatory carcinoma and the presence of recurrent breast abscesses, histopathological diagnosis being indispensable. In this report, we present the case of a patient with idiopathic granulomatous mastitis, a rare condition seen in Brazil.

\section{CASE REPORT}

Female patient, 32 years old, smoker, without other comorbidities, using only quarterly injectable contraceptives. She sought medical attention due to a painful nodule in the left breast, which was rapidly growing. She denied history of trauma and family history of breast cancer. Breast ultrasound was performed, which is a possible mastitis process, with antibiotics and analgesia being prescribed. Required to remain in the condition, after instituted treatment, it was decided to perform a new image exam in another service, with a report showing a heterogeneous area, predominantly hypoechoic, oval, consistent with BIRADS 4A. Two incisional biopsies were performed at different times, which revealed chronic granulomatous mastitis, negative for neoplasia. A search for fungi, acid-resistant bacilli (ARB) and bacteria was negative. The patient evolved with recurrent abscesses, requiring surgical drainage. Treated bulletin with prednisone in immunosuppressive dose was changed after methotrexate and reduced to corticosteroid dose. After adverse effects of this medication, it was changed to azathioprine, which remains until now.

\section{CONCLUSION}

Idiopathic granulomatous mastitis is a rare chronic inflammatory disease of unknown etiology, which occurs mainly in young women. The clinical, radiological and ultrasound characteristics are similar to those of breast cancer, which can confuse the diagnosis. The ultrasound findings of multiple hypoechoic, contiguous and confluent tubular images in young women common to this diagnosis. 